\title{
Diabetes $\mathbb{\&}$ its Complications
}

\section{New Horizons for the Ophthalmic Treatment of Diabetic Retinopathy and Diabetic Macular Edema Post the Anti-VEGF Era}

\author{
Richard R. Sadig*, Hassan Lami and Daniel Moawad
}

Faculty of Medicine, University of Notre Dame Sydney, 160 Oxford St, Darlinghurst NSW 2010, Australia.
*Correspondence:

Richard R. Sadig, Faculty of Medicine, University of Notre Dame Sydney, 160 Oxford St, Darlinghurst NSW 2010, Australia, Tel: +61 2 95945671; E-mail: richard.sadig1@my.nd.edu.au.

Received: 20 August 2017; Accepted: 24 August 2017

Citation: Richard R. Sadig, Hassan Lami, Daniel Moawad. New Horizons for the Ophthalmic Treatment of Diabetic Retinopathy and Diabetic Macular Edema Post the Anti-VEGF Era. Diabetes Complications. 2017; 1(2) 002; 1-2.

Whilst anti-vascular endothelial growth factor (VEGF) treatments have been quite revolutionary in preventing eye blindness due to diabetic retinopathy (DR), a small subset of patients do not seem to respond to traditional anti-VEGF injections, in addition to other practical issues such as adherence. Consequently a novel hunt for new targets that could alleviate the effects of diabetic retinopathy and diabetic macular edema (DME) are currently sought after.

The first of these alternative targets appears to be through the targeting of the bradykinin pathway in the body. The action of bradykinin and kallidin on the bradykinin-2 receptors (B2R) causes vasodilation of blood vessels [via the kinin-kallikrein system (KKS)]. Kallikriens are noted to be upregulated in the eyes of type 1 diabetic mice, and in humans, plasma prekallikrein was elevated in both type I and type II diabetic patients. This was shown quite convincingly when intravitreal injections of plasma kallikrein in healthy human eyes showed increased retinal vascular permeability. The vasogenic effects were reversed with the systemic administration of a plasma kallikrein inhibitor, showing potential promise in not only the development of non-VEGF injectables for DR, but the promise for an oral treatment for DR without the risk of endophthalmitis. Whilst clinical trials have already begun for the use of kallikrein inhibitors in systemic hereditary angioedemas, none of them yet have focussed their indication for DR or diabetic macular edema (DME) $[1,2]$.

The second of the new potential targets work on a similar system to the KKS system but this time on the arm of the renin-angiotensin system (RAAS). RAAS has a similar theoretical basis to the KKS in the sense that it aims to target vessel vascular tone with a greater focus on vascular stability than through its vasodilatory or anti-hypertensive properties. There is however speculation that the ACE inhibitor mediated effects of DR non -progression may also be through the inhibition of the KKS system (given the two are intimately related). The benefits of utilising an ACE inhibitor approach to DR stabilisation is that this class of drugs are quite familiar in clinical world of medicine and its side effects are well documented. More experience is needed however for the KKS targeting drugs $[1,3]$.

The last of these interrelated drug approaches to DR seems to be the targeting of various vascular growth factors which either may favour vascular stabilisation or angiogenesis. One such implicated family of growth factors include the "angiopoietins"- consisting primarily of angiopoietin-1 (ang-1) and angiopoietin-2 (ang2). Ang-2, in the presence of VEGF, leads to angiogenesis and is elevated in the vitreous of poorly controlled diabetics. On the converse, Ang-1 seems to be involved with anti-angiogenesis, vascular stabilisation and anti-adhesion effects, conferring what seems to be vascular protection against diabetic retinopathy. The two paradigms of treatment would be to either facilitate the blockade of Ang-2 using a monoclonal antibody, or to directly inject Ang-1 factors to aid in the stabilisation of retinal vessels $[1,4]$.

Whilst the angiopoietins and anti-VEGF seem like desirable methods to inhibit angiogenesis, they still rely on regular injections to the eyes by medical practitioners - which is both cumbersome, costly and carries the rare risk of endophthalmitis [5]. All 'eyes' are now turned toward the combined RAAS/KKS system inhibitors for a non-invasive method to control DR progression using orally available routes; which are both familiar to medical practitioners and widely available due to their lower comparative cost. Adherence to ACE medication could improve as ACE inhibitors are already used for other common indications such as hypertension, and may not require frequent specialist appointments. 


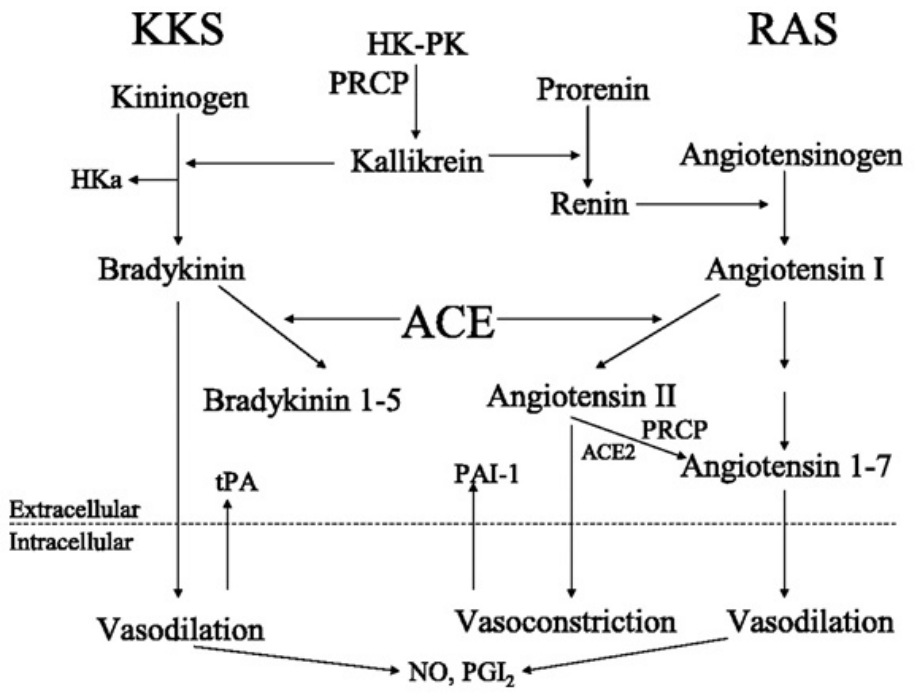

Figure 2: The complex interplay between KKS and RAAS (6).

\section{References}

1. Bolinger MT, Antonetti DA. Moving Past Anti-VEGF: Novel Therapies for Treating Diabetic Retinopathy. International Journal of Molecular Sciences. 2016; 17: 1498.

2. Kita T, Clermont AC, Murugesan N, et al. Plasma KallikreinKinin System as a VEGF-Independent Mediator of Diabetic Macular Edema. Diabetes. 2015; 64: 3588-3599.

3. Mauer M, Zinman B, Gardiner R, et al. Renal and Retinal Effects of Enalapril and Losartan in Type 1 Diabetes. The New England journal of medicine. 2009; 361: 40-51.

4. Gamble JR, Drew J, Trezise L, et al. Angiopoietin-1 Is an Antipermeability and Anti-Inflammatory Agent In Vitro and

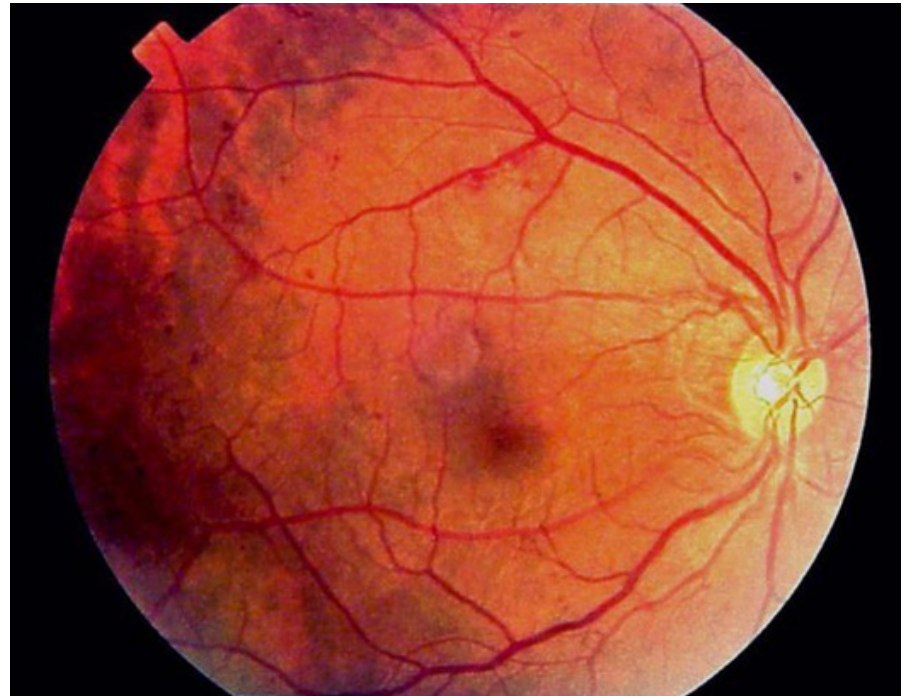

Figure 2: Fundoscopic view of early diabetic retinopathy (7).

Targets Cell Junctions. Circulation Research. 2000; 87: 603.

5. Antonetti DA, VanGuilder HD, Mao-Lin C. Vascular Permeability in Diabetic Retinopathy. In: Duh EJ, editor. Diabetic Retinopathy. Totowa, NJ: Humana Press; 2008. 333352.

6. Schmaier AH. The kallikrein-kinin and the renin-angiotensin systems have a multilayered interaction. American Journal of Physiology - Regulatory, Integrative and Comparative Physiology. 2003; 285: R1.

7. Bhavsar A. Diabetic Retinopathy: Practice Essentials, Pathophysiology, Etiology [Internet]. Emedicine.medscape. com. 2017. 